\title{
Carcinoma corticoadrenal, reporte de caso
}

\author{
Adrenocortical carcinoma, case report
}

Yeniseik D. Cortés-Vázquez ${ }^{*}$, Laura C. Mejía-Rios ${ }^{1}$, Alejandro Priego-Niño ${ }^{1}$, Víctor Salgado-Arroyo', Héctor E. Cabrales-Santiago², Claudia Gutiérrez-Quiróz ${ }^{2}$ y Nallely Ochoa Cruz ${ }^{2}$

${ }^{1}$ Servicio de Urología; ${ }^{2}$ Servicio de Patología. Unidad Médica de Alta Especialidad Manuel Ávila Camacho, Instituto Mexicano del Seguro Social, Puebla, Puebla México

\section{Resumen}

El carcinoma corticoadrenal es una neoplasia rara, altamente agresiva, de distribución bimodal, con predominio en el sexo femenino, de la cual el $20 \%$ de los casos se diagnostican de manera incidental. Se presenta el caso de un varón de 43 años con dolor de tipo cólico en el flanco izquierdo, pérdida de peso y fiebre intermitente. La tomografía computarizada mostró un tumor en la glándula suprarrenal izquierda y metástasis hepática. Se realizó adrenalectomía radical en bloque, con reporte anatomopatológico de carcinoma corticoadrenal, Weiss de 7 puntos y Ki67 40\%. El carcinoma corticoadrenal es una neoplasia agresiva y de presentación clínica variable. La terapia sistémica es importante incluso en pacientes con enfermedad localizada e independientemente de la cirugía.

Palabras clave: Carcinoma corticoadrenal. Puntaje Weiss. Suprarrenal.

\section{Abstract}

The adrenocortical carcinoma is rare and aggressive. It has a bimodal presentation, predominantly female, > $20 \%$ of cases will be diagnosed incidentally. 43-year-old male, with colic pain in the left flank, weight loss and intermittent fever. Computed tomography with a tumor on the left adrenal with liver metastases, block resection surgery was performed, pathological report of adrenocortical carcinoma with a 7 points of Weiss score and Ki67 40\%. Adrenocortical carcinoma is a rare and aggressive neoplasm; the clinical presentation is variable. Systemic therapy is important even in patients with localized disease and independent of surgical approach.

Key words: Adrenocortical carcinoma. Weiss score. Adrenal.

\section{Introducción}

Los tumores suprarrenales son comunes y afectan al $3-10 \%$ de la población ${ }^{1,2}$. El 1-4\% de los casos se presentan como incidentalomas y la mayoría de estos (hasta el 80\%) corresponden a adenomas; solo el $8 \%$ de los casos se presentan como carcinomas².

El carcinoma corticoadrenal (CCA) es una enfermedad rara y muy agresiva, con una incidencia estimada de 0.5-2 casos por millón de personas/año y una

\section{Correspondencia:}

*Yeniseik D. Cortés-Vázquez

2 Norte 2004

Col. Centro

Fecha de recepción: 24-06-2020

C.P. 72000 , Puebla, Pue., México

E-mail: delhicortes@gmail.com
Cir Cir. 2021;89(5):664-668

Contents available at PubMed www.cirugiaycirujanos.com 0009-7411/@ 2020 Academia Mexicana de Cirugía. Publicado por Permanyer. Este es un artículo open access bajo la licencia CC BY-NC-ND (http://creativecommons.org/licenses/by-nc-nd/4.0/). 
prevalencia mundial de 4-12 casos por millón de personas/año ${ }^{3}$. Presenta una distribución bimodal, con un primer pico en niños menores de 10 años y un segundo pico durante la cuarta y quinta décadas de la vida, con un ligero predominio en las mujeres, que representan hasta el $60 \%$ de los casos'.

La enfermedad metastásica estará presente al inicio del diagnóstico en un $50 \%$ de los pacientes, y en el otro $50 \%$ se presentará como progresión o recurrencia durante el seguimiento a corto plazo. Los sitios habituales de metástasis son el hígado y los pulmones principalmente, y con menor menos frecuencia el hueso y el peritoneo. No existen criterios absolutos de malignidad para las lesiones confinadas a la glándula suprarrenal, pero las características histológicas y un puntaje de Weiss $\geq 3$ generalmente son indicadores de malignidad, así como el índice de proliferación Ki-67 > 20\% .

\section{Caso clínico}

Varón de 43 años con diabetes mellitus tipo 2 en adecuado control. Inició con dolor de tipo cólico en el hipocondrio izquierdo, que se irradiaba a la región lumbar homolateral, con una puntuación en la escala visual análoga de $8 / 10$, fiebre intermitente y pérdida de peso no intencionada de $10 \mathrm{~kg}$ en 3 meses. La tomografía de abdomen contrastada evidenció un tumor dependiente de la glándula suprarrenal izquierda, de $10 \times 12 \mathrm{~cm}$, de 91 unidades Hounsfield (UH) y realce a $167 \mathrm{UH}$ tras la aplicación de medio de contraste, que desplazaba las estructuras adyacentes, así como lesiones hepáticas en los segmentos VII y VIII con realce tras la aplicación de medio de contraste (Fig. 1). Las catecolaminas y las metanefrinas plasmáticas y urinarias fueron normales. Se realizó una adrenalectomía radical en bloque (Fig. 2), sin complicaciones transoperatorias, con un sangrado cuantificado en $1300 \mathrm{ml}$, adecuada evolución posoperatoria y egreso domiciliario al cuarto día. El reporte histopatológico fue de carcinoma corticoadrenal de alto grado, de $16 \mathrm{~cm}$ diámetro, con necrosis extensa (70\%) que invade la cápsula, con presencia de invasión linfovascular focal, márgenes quirúrgicos libres de neoplasia, sin invasión a estructuras adyacentes, y con índice mitótico de 25 (Fig. 3). En la inmunohistoquímica, inhibina, Melan-A y Ki-67 (40\%) fueron positivas; sinaptofisina, cromogranina y $\mathrm{S} 100$, negativas (Fig. 4). El paciente se encuentra actualmente en protocolo para inicio de terapia sistémica.

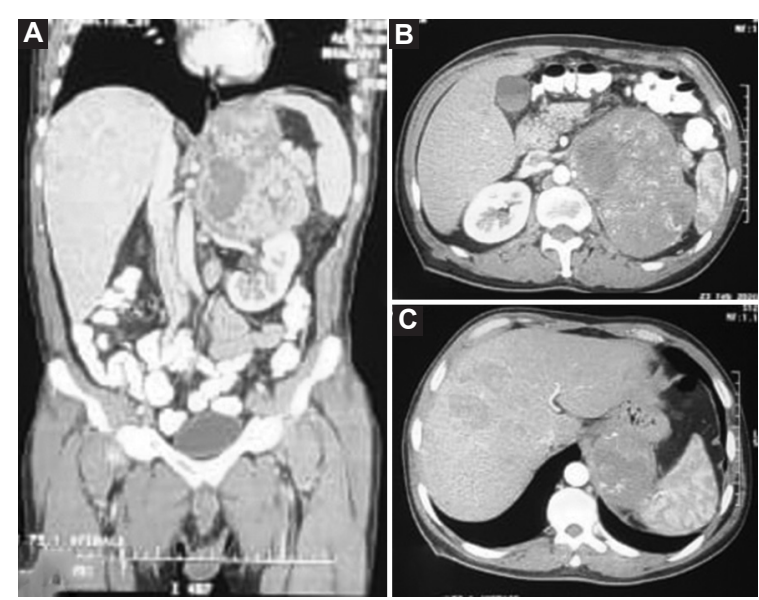

Figura 1. Tomografía computarizada contrastada. A: corte coronal que muestra un tumor de suprarrenal izquierda de $10 \times 12 \mathrm{~cm}$, de 91 UH, con realce a $167 \mathrm{UH}$. B-C: corte axial que muestra un tumor de suprarrenal izquierda con desplazamiento del riñón y del bazo.

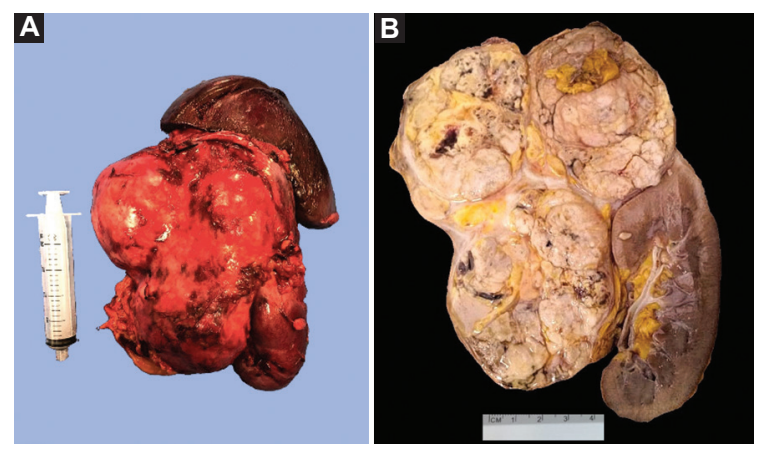

Figura 2. A: pieza quirúrgica de resección en bloque por actividad tumoral. B: apariencia macroscópica: tumor de gran tamaño, sólido y con extensas áreas de necrosis, que comprime el polo superior renal.

\section{Discusión}

Los tumores suprarrenales se presentan en su mayoría como tumores benignos, principalmente adenomas, hasta en el $80 \%$ de los casos ${ }^{1}$. Los tumores malignos son raros, con principal importancia el CCA por su agresividad, ya que la sobrevida depende de la etapa al momento del diagnóstico, alcanzando en las etapas I y II una sobrevida del $60-80 \%$ a los 5 años, en estadio III o localmente avanzado una sobrevida a 5 años del $35-50 \%$, y en estadio IV una sobrevida a 5 años del $13 \% \%^{3,4}$.

Los CCA pueden ser tumores funcionales en un 40$60 \%$ de los $\operatorname{casos}^{5}$, y pueden producir diferentes síndromes clínicos de acuerdo con la sobreproducción hormonal involucrada ${ }^{5}$. Así, pueden presentarse con elevación de cortisol (síndrome de Cushing) en un 


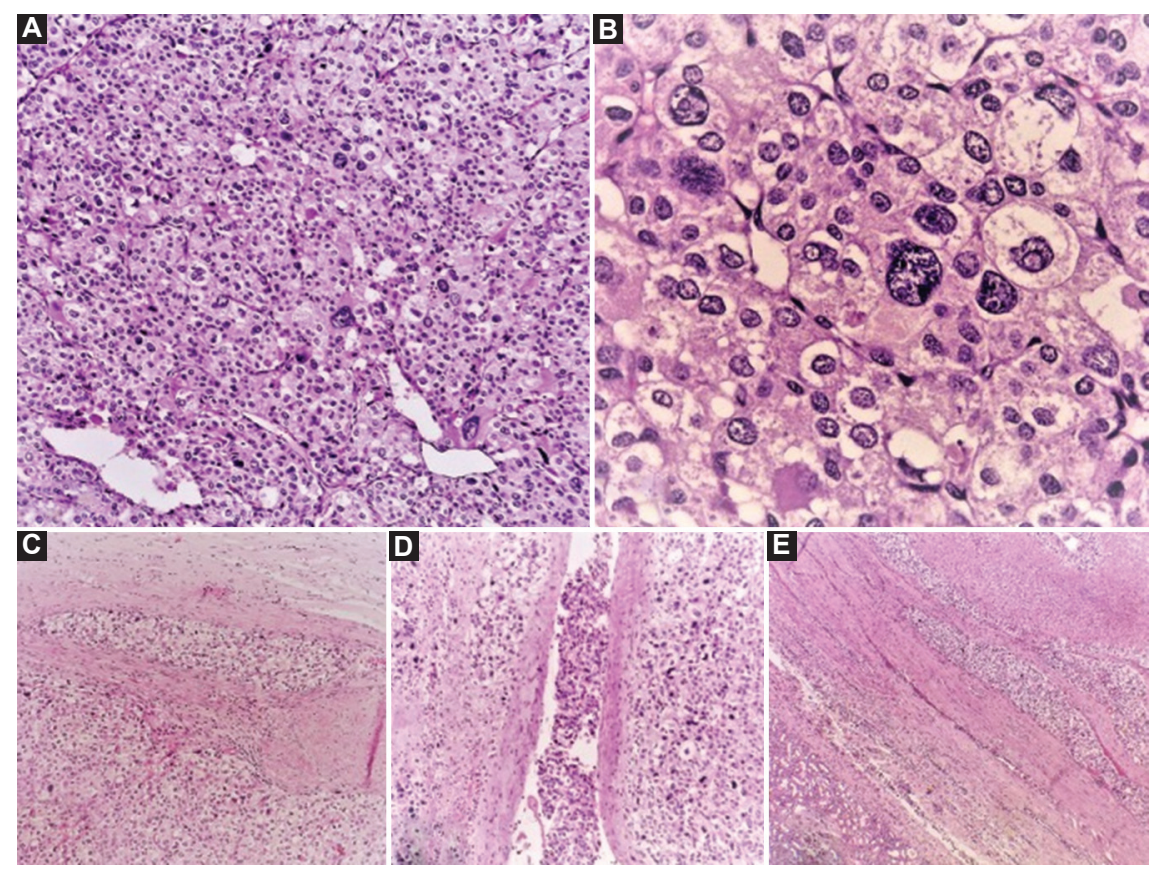

Figura 3. Carcinoma corticoadrenal. A: patrón de crecimiento sólido con heterogeneidad celular. B: a mayor aumento se observan nucleomegalia, cromatina granular gruesa con distribución irregular e inclusiones intranucleares. C: invasión a la cápsula adrenal. D: invasión vascular. E: tumor y riñón subyacente sin invasión (tinción de hematoxilina y eosina).

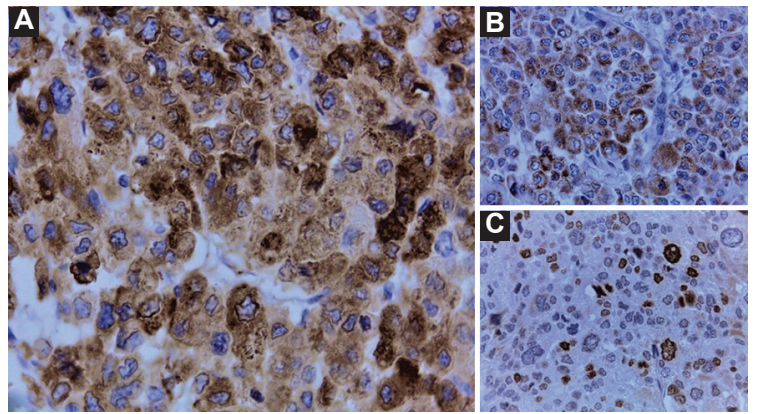

Figura 4. Inmunohistoquímica de carcinoma corticoadrenal. A: inhibina positiva. B: Melan-A positiva. C: índice de proliferación celular (Ki-67), 40\% nuclear.

50-70\%, elevación de andrógenos (virilización) 20-30\%, elevación de estrógenos (feminización) $5 \%$ y elevación de mineralocorticoides en 2-3\%. En aproximadamente un $30-40 \%$ de los casos, los síntomas se deben al tamaño del tumor, que produce efecto de masa sobre los órganos adyacentes, causando dolor abdominal, distensión abdominal, sensación de plenitud, náusea 0 vómito. La mayoría de los CCA son esporádicos, pero también se han asociado a algunos trastornos hereditarios, como el síndrome de Li-Fraumeni, el síndrome de Beckwith-Wiedemann, el síndrome de Lynch y la neoplasia endocrina múltiple de tipo 1 y poliposis adenomatosa familiar ${ }^{6}$.

En el abordaje diagnóstico se debe realizar un panel metabólico que incluya mineralocorticoides, glucocorticoides, andrógenos y catecolaminas, que permitirá diferenciar entre CCA y otros tumores como el feocromocitoma; en los CCA es común la elevación de los andrógenos y del cortisol. En un estudio realizado por Sada, et al. ${ }^{7}$, el $52.6 \%$ correspondieron a tumores funcionales, de los cuales el $47.1 \%$ se presentaron con sobreproducción de cortisol, el $25.1 \%$ con hormonas combinadas, el $19.3 \%$ con hormonas sexuales y el $7.9 \%$ con aldosterona; resultó estadísticamente significativo $(p=0.001)$ el estadio clínico avanzado (IV) como único factor independiente para tumores funcionales, con un $41.4 \%$, frente a un $21.4 \%$ para los no funcionales.

En el abordaje diagnóstico tienen un papel importante los estudios de imagen, ya que otorgan información que puede orientar hacia el comportamiento de tumores malignos, por lo que hay que recordar que habitualmente los CCA se presentan como tumores unilaterales, de dimensiones $>6 \mathrm{~cm}$ en un $90 \%$ de los $\operatorname{casos}^{5}$ y con áreas de necrosis. En la tomografía computarizada se caracterizan por ser tumores con densidad > $10 \mathrm{UH}$, con una sensibilidad 
del $93-100 \%$ y una especificidad del $71-72 \% \%^{2,6,8}$, con lavado lento del contraste $(<50 \%)^{8}$; también puede haber calcificaciones y en ocasiones un trombo en la vena renal o la vena cava. En la resonancia magnética con gadolinio, en T2 se observan lesiones hiperintensas en comparación con el hígado y heterogéneas $^{8,9}$. La tomografía por emisión de positrones con 18-fluorodesoxiglucosa (18-FDG) puede tener utilidad en el seguimiento de los pacientes 0 en la identificación de lesiones a distancia, pero es de especificidad limitada por la absorción de la 18FDG por la suprarrenal benigna y los feocromocitomas. Actualmente es prometedor el uso de 11C-metomidato como marcador, el cual puede diferenciar entre adenomas y CCA, por lo que sería de mayor utilidad en el estudio de los tumores adrenales; sin embargo, aún hacen falta más estudios para demostrarlo ${ }^{10}$.

Existen factores pronósticos que pueden predecir la sobrevida del paciente, como el índice de proliferación Ki-67 > 20\% y la puntuación de Weiss (grado nuclear, índice de mitosis en campo de alta frecuencia, figuras mitóticas atípicas, células claras $<25 \%$, arquitectura difusa $>33 \%$ del tumor, necrosis, invasión vascular, invasión sinusoidal, invasión capsular) $>6$, que se han asociado a un pronóstico pobre ${ }^{11-13}$. Los predictores más importantes de supervivencia en estos pacientes son el grado tumoral, el estadio tumoral y la resección quirúrgica ${ }^{13}$.

En el diagnostico histopatológico desempeña un papel importante la inmunohistoquímica para confirmar el origen adrenocortical. Existen diferentes biomarcadores que pueden plantear el diagnóstico, por lo que es importante conocer la especificidad y la sensibilidad de cada uno. El factor esteroidogénico 1 se considera el más confiable y específico, ya que se expresa en la corteza suprarrenal no tumoral y en neoplasias corticales, considerándose distintivo de tejidos esteroidogénicos; tiene una especificidad del $100 \%$ y una sensibilidad del $98-100 \%{ }^{14}$. Melan-A se ha descrito en tejido esteroidogénico, con una sensibilidad y una especificidad superiores al $95 \%$. Exiten otros biomarcadores, como inhibina alfa, calretinina y D2-40, que se pueden expresar en tumores adrenales y de celulas mesoteliales. Ki-67, como ya se ha mencionado, tiene valor pronóstico para el diagnóstico y la sobrevida. La cromogranina A suele ser negativa en el CCA y positiva en el feocromocitoma.

Se ha propuesto la clasificación de la Red Europea para el Estudio de Tumores Suprarrenales $(\text { ENSAT })^{6,13}$, un sistema de estadificación que, al igual que las guías del National Comprehensive Cancer Network (NCCN), indica que en los estadios I y II son tumores localizados con un tamaño de $\leq 50>5 \mathrm{~cm}$, respectivamente; el estadio III se define por la presencia de ganglios linfáticos positivos, infiltración del tejido circundante o trombo tumoral en la vena cava o la vena renal; y finalmente, el estadio IV corresponde a metástasis a distancia.

En la actualidad, el único enfoque curativo para el CCA es la resección tumoral completa ${ }^{3}$, por lo que incluso en la enfermedad metastásica sigue siendo una opción terapeútica para mejorar la sobrevida cuando sea posible la resección completa. Las terapias adyuvantes tienen como objetivo disminuir la posibilidad de recurrencia ${ }^{4}$. Es importante recordar que el uso de terapias adyuvantes es importante incluso en la enfermedad localizada.

El mitotano es un fármaco adrenocitolítico aprobado por la Food and Drug Administration y por la Agencia Europea de Medicamentos para el tratamiento del CCA. Se requieren unas concentraciones plasmáticas > $14 \mathrm{mg} / /^{15}$, y los efectos secundarios debido a su toxicidad hacen que sea dificil su manejo. Se han estudiado otros esquemas terapéuticos, pero no se ha logrado sustituir el manejo con mitotano.

Un estudio retrospectivo de 177 pacientes realizado por Terzolo, et al. ${ }^{16}$, en el que 47 pacientes recibieron terapia adyuvante y se compararon con 55 y 130 (55 grupo 1 y 75 grupo 2) sin terapia adyuvante, sugiere que el uso de mitotano posterior a la resección radical del CCA prolonga significativamente la sobrevida libre de recurrencia a 42 meses, frente a 10 meses en el grupo control 1 y 25 meses en el grupo control 2.

El ensayo FIRM-ACT, realizado por Fassnacht, et al. ${ }^{17}$, incluyó 304 pacientes con CCA metastásico y comparó la asociación de mitotano con etopósido, cisplatino y doxorubicina (M-EDP) frente a mitotano y estreptozotocina (M-Sz) como tratamiento de primera o segunda línea, obteniendo una mejor supervivencia libre de progresión con M-EDP: 5.0 meses frente a 2.1 meses con M-Sz $(p<0.001)$.

En nuestro paciente, sin evidencia clínica ni bioquímica de funcionalidad, que debutó como enfermedad avanzada con un estadio clínico IV, con actividad metastásica hepática, fue necesaria la resección en bloque, obteniendo unos márgenes negativos en la pieza quirúrgica. Aun así, es necesaria terapia sistémica adyuvante. Sin embargo, el pronóstico es malo 
debido por su puntuación de Weiss de 7 puntos, el Ki-67 del $40 \%$ y el estadio clínico IV, por lo que la probabilidad de sobrevivir 5 años será menor del 13\%.

\section{Conclusiones}

El CCA es una neoplasia rara y agresiva, con una presentación clínica variable. Aunque suele diagnosticarse de manera incidental, en la mayoría de los casos se presenta como enfermedad avanzada al momento del diagnóstico. En el estudio histopatológico es importante la realización de inmunohistoquímica y de índices de proliferación y mitóticos, ya que ayudan a establecer el pronóstico y a diferenciar tumores neuroendocrinos distintos del CCA. La resección quirúrgica sigue siento el tratamiento estándar y con mejor sobrevida, sin olvidar que la adyuvancia con mitotano incluso en pacientes sin metástasis es una opción terapéutica que mejora la sobrevida.

\section{Agradecimientos}

Los autores agradecen a la institución y a sus maestros.

\section{Conflicto de intereses}

Los autores declaran no tener conflictos de intereses.

\section{Responsabilidades éticas}

Protección de personas y animales. Los autores declaran que para esta investigación no se han realizado experimentos en seres humanos ni en animales.

Confidencialidad de los datos. Los autores declaran que han seguido los protocolos de su centro de trabajo sobre la publicación de datos de pacientes.
Derecho a la privacidad y consentimiento informado. Los autores han obtenido el consentimiento informado de los pacientes y/o sujetos referidos en el artículo. Este documento obra en poder del autor de correspondencia.

\section{Bibliografía}

1. Libè R, Fratticci A, Bertherat J. Adrenocortical cancer: pathophysiology and clinical management. Endocr Relat Cancer. 2007;14:13-28.

2. Else T, Kim AC, Sabolch A, Raymond VM, Kandathil A, Caoili EM, et al. Adrenocortical carcinoma. Endocr Rev. 2014;35:282卬326.

3. Almeida MQ, Bezerra-Neto JE, Mendonça BB, Latronico AC, Fragoso MCBV. Primary malignant tumors of the adrenal glands. Clinics (Sao Paulo). 2018;73:756-63.

4. Fassnacht M, Johanssen S, Quinkler M, Bucsky P, Willenberg HS, Beuschlein $F$, et al. Limited prognostic value of the 2004 International Union Against Cancer staging classification for adrenocortical carcinoma: proposal for a Revised TNM Classification. Cancer. 2009;115:243050.

5. Ahmed AA, Thomas AJ, Ganeshan DM, Blair K, Lall C, Lee JT, et al. Adrenal cortical carcinoma: pathology, genomics, prognosis, imaging features, and mimics with impact on management. Abdom Radiol (NY). 2020;45:945ロ63.

6. Gaujoux S, Mihai R; Joint Working Group of ESES and ENSAT. European Society of Endocrine Surgeons (ESES) and European Network for the Study of Adrenal Tumours (ENSAT) recommendations for the surgical management of adrenocortical carcinoma. Br J Surg. 2017;104:358076.

7. Sada A, Asaad M, Bews KA, Thompson GB, Young WF Jr, Bancos I, et al. Comparison between functional and non-functional adrenocortical carcinoma. Surgery. 2020;167:216-23.

8. Íñiguez-Ariza NM, Kohlenberg JD, Delivanis DA, Hartman RP, Dean DS, Thomas MA, et al. Clinical, biochemical, and radiological characteristics of a single-center retrospective cohort of 705 large adrenal tumors. Mayo Clin Proc Innov Qual Outcomes. 2017;2:30ロ9.

9. Bharwani N, Rockall AG, Sahdev A, Gueorguiev M, Drake W, Grossman $A B$, et al. Adrenocortical carcinoma: the range of appearances on CT and MRI. AJR Am J Roentgenol. 2011;196:706ロ14.

10. Khan TS, Sundin A, Juhlin C, Långström B, Bergström M, Eriksson B. 11C-metomidate PET imaging of adrenocortical cancer. Eur J Nucl Med Mol Imaging. 2003:30:403-10.

11. Vaidya A, Nehs M, Kilbridge K. Treatment of adrenocortical carcinoma. Surg Pathol Clin. 2019;12:99701006.

12. Liang J, Liu Z, Zhou L, Tang Y, Zhou C, Wu K, et al. The clinical utility of 'GRAS' parameters in stage I-III adrenocortical carcinomas: long-term data from a high-volume institution. Endocrine. 2020;67:449-56.

13. Kebebew E, Reiff E, Duh QY, Clark OH, McMillan A. Extent of disease at presentation and outcome for adrenocortical carcinoma: have we made progress? World J Surg. 2006;30:87208.

14. Mete O, Asa SL, Giordano TJ, Popotti M, Sasano H, Volante M. Immunohistochemical biomarkers of adrenal cortical neoplasms. Endocr Pathol. 2018;29:137-49.

15. Paragliola RM, Torino F, Papi G, Locantore P, Pontecorvi A, Corsello SM Role of mitotane in adrenocortical carcinoma-review and state of the art. Eur Endocrinol. 2018;14:62ロ6.

16. Terzolo M, Angeli A, Fassnacht M, Daffara F, Tauchmanova L, Conton PA, et al. Adjuvant mitotane treatment for adrenocortical carcinoma. N Engl J Med. 2007:356:2372ロ80.

17. Fassnacht M, Terzolo M, Allolio B, Baudin E, Haak H, Berruti A, et al. Combination chemotherapy in advanced adrenocortical carcinoma. $\mathrm{N}$ Engl J Med. 2012;366:2189u97. 